\title{
DEVELOPMENT OF A METHOD FOR ASSESSMENT AND FORECASTING OF THE RADIO ELECTRONIC ENVIRONMENT
}

\author{
Oleg Sova \\ Department of Automated Control Systems ${ }^{1}$ \\ Andrii Shyshatskyi $\bowtie$ \\ Research Department of Electronic Warfare Development \\ Central Scientifically-Research Institute of Arming and Military Equipment \\ of the Armed Forces of Ukraine \\ 28 Povitrofloski ave., Kyiv, Ukraine, 03049 \\ ierikon13@gmail.com \\ Olha Salnikova \\ Educational and Research Center of Strategic Communications in the Sphere \\ of National Security and Defense \\ National Defense University of Ukraine named after Ivan Cherniakhovskyi \\ 28 Povitrofloski ave., Kyiv, Ukraine, 03049 \\ Oleksandr Zhuk \\ Department of Military Training ${ }^{l}$ \\ Oleksandr Trotsko \\ Department of Automated Control Systems ${ }^{1}$ \\ Yaroslav Hrokholskyi \\ Department of Automated Control Systems ${ }^{1}$ \\ ${ }^{1}$ Military Institute of Telecommunications and Information Technologies \\ named after Heroes of Kruty \\ 45/1 Moskovska str., Kyiv, Ukraine, 01011
}

Corresponding author

Abstract

Decision making support systems (DSS) are actively used in all spheres of human life. The system of the electronic environment analysis is not an exception. However, there are a number of problems in the analysis of the electronic environment, for example: the signals are analyzed in a complex electronic environment against the background of intentional and natural interference. Input signals do not match the standards, and their interpretation depends on the experience of the operator (expert), the completeness of additional information on a particular task (uncertainty condition). The best solution in this situation is found in the integration with the data of the information system analysis of the electronic environment, artificial neural networks and fuzzy cognitive models. Their advantages are also the ability to work in real time and quick adaptation to specific situations. The article develops a method for assessing and forecasting the electronic environment.

Improving the efficiency of evaluation information processing is achieved through the use of evolving neuro-fuzzy artificial neural networks; learning not only the synaptic weights of the artificial neural network, the type and parameters of the membership function. The efficiency of information processing is also achieved through training in the architecture of artificial neural networks; taking into account the type of uncertainty of the information that has to be assessed; synthesis of rational structure of fuzzy cognitive model. It reduces the computational complexity of decision-making; has no accumulation of learning error of artificial neural networks as a result of processing the information coming to the input of artificial neural networks. The example of assessing the state of the electronic environment showed an increase in the efficiency of assessment at the level of 15-25\% on the efficiency of information processing.

Keywords: artificial intelligence, electronic environment, intelligent systems, decision making support systems. 


\section{Introduction}

Decision making support systems (DMSS) are actively used in all spheres of human life. The creation of intelligent DMSS has become a natural continuation of the widespread use of the classical type DMSS. Intelligent DMSS have been widely used to solve specific military tasks [1, 2]:

- planning the deployment, operation of communication systems and data transmission;

- automation of troops and weapons control;

- collection, processing and generalization of intelligence information about the intelligence objects state, etc.

One of such tasks for using intelligent DMSS is the analysis and forecasting of the electronic situation, which is also reduced to a multi-criteria analysis of alternatives, given the large number and diversity of troops (forces) groups. From a practical point of view, the efficiency of the use of radio frequency resources and the efficiency of radio communication systems (electronic suppression) depend on the efficiency of analysis and forecasting of the electronic environment. However, while analyzing the tasks of the radio electronic environment (REE) there is a number of problematic issues, for example:

1. Signals are analyzed in a complex electronic environment against the background of different origins interference.

2. The input signals do not match the standards due to the influence of different interference types.

3. Interpretation of signals depends on the experience of the operator (expert), the completeness of additional information on a specific task (conditions of uncertainty).

\section{Materials and methods of research}

The work [3] reveals the essence of cognitive modeling and scenario planning. The system of complementary principles of construction and realization of scenarios is offered, various approaches to construction of scenarios are allocated, the procedure of scenarios modeling on the basis of fuzzy cognitive maps is described. It is proposed to identify the concepts of the cognitive map based on the analysis of the internal and external environment of the organization, which will systematically look at the business conditions of the enterprise, predict further development and make the right management decisions. The approach proposed by the authors does not take into account the type of uncertainty about the analysis object state and does not take into account the delay in processing data about the state of the object.

The work [4] conducts the analysis of the main approaches for cognitive modeling. Cognitive analysis allows to: explore problems with fuzzy factors and relationships; take into account changes in the external environment and use objectively formed trends in the situation to their advantage. It is noted that it is necessary to develop a system of criteria for the possibility of formalization and automation of decision-making in problem areas. It is also stated that the objectivity of the processed information must be taken into account.

The work [5] describes the agent-based approach used in a multi-agent information-analytical system and the problems of decision-making support system are considered. The disadvantages of this approach include the limited representation of complex systems, for example, none of the agents has an idea of the whole system.

The work [6] presents the method of large data sets analysis. This method is focused on finding hidden information in large data sets. The method includes operations of generating analytical baselines, reducing variables, detecting sparse features and specifying rules. The disadvantages of this method include the inability to take into account different decision evaluation strategies.

The work [7] proposes an approach for estimating the client living cost in the field of air transportation. In this approach, a regression model is used first, followed by an indirect estimation model. At the final stage, the evaluation results are compared using both evaluation models. The disadvantages of this approach include the inability to determine the adequacy of the assessment.

The work [8] proposes a complex method of processing various data types in intelligent DMSS. This approach is based on the concept of a data lake. This approach allows to process 
both structured and unstructured data. The disadvantages of this approach include the inability to determine the adequacy of the assessment and to establish the impact of each indicator on each other.

The vast majority of known intelligent DMSS use methods of multi-criteria fuzzy alternatives evaluation. However, the analysis of works [1, 3-13] showed that in the vast majority of these methods have the following common limitations:

- complexity of forming a multilevel evaluation structure;

- lack of the compatibility consideration of unevenly significant indicators;

- lack of possibility to jointly perform direct and inverse evaluation tasks with the support of choosing the best solutions;

- lack of possibility to form a generalized evaluation indicator and the choice of solutions based on sets of partial indicators, which change taking into account the complex multi-level structure of evaluation;

- complexity of aggregating heterogeneous indicators (both quantitative and qualitative) of evaluation and selection of solutions that differ in measuring scales and ranges of values;

- failure to take into account different decision making evaluation strategies;

- complexity of setting up (adaptation) of evaluation models while adding (excluding) indicators and changing their parameters (compatibility and significance of indicators);

- not taking into account the type of uncertainty of the initial data about the object state.

To achieve this aim, the following objectives are set:

- to carry out the mathematical formulation of the problem of developing methods for RES assessing and forecasting in intelligent decision making support systems;

- to develop a methodology for assessing and forecasting RES in intelligent decision making support systems;

- to evaluate the effectiveness of the proposed method.

In developing a methodology for RES assessing and forecasting in intelligent decision making support systems used the following methods:

- fuzzy temporal models - to establish mutual relations between indicators and determine the degree of indicators influence on each other to solve the problem of RES analysis and forecasting;

- evolving artificial neural networks - for training and adjusting the knowledge bases of the electronic environment. For the training of evolving artificial neural networks, the method of training artificial neural networks developed in previous works was used, which allows to perform the in-depth training of artificial neural networks, which consists in learning the architecture, type and parameters of the membership function.

RES modeling estimation and forecasting methods in intelligent decision making support systems was performed with the following parameters:

- radio devices with pseudo-random adjustment of the operating frequency. Frequency range is $30-512 \mathrm{MHz}$; transmitter power is $10 \mathrm{~W}$; radiated frequency bandwidth is $12.5 \mathrm{kHz}$, receiver sensitivity is $120 \mathrm{~dB}$; the number of radio communication devices (RCD) in the network is 5 . The number of frequency channels for radio reconfiguration (RR) is 10000; the number of adjustments is from 333.5 to 1000 jumps/sec;

- radio electronic suppression (RES) complexes is 2 . The frequency range of RES complexes is $30-2000 \mathrm{MHz}$; transmitter power is $2000 \mathrm{~W}$; the maximum frequency band that can be suppressed simultaneously is $80 \mathrm{MHz}$. The type of interference of RES complexes is noise obstruction interference with frequency manipulation, as one of the most common and the influence of which is well known; the strategy of the RES complex is dynamic.

The simulation was performed using MathCad 2014 software and an Intel Core i3 PC. 5 programmable LimeSDR transceivers (USA) with GNU Radio software (Germany) were connected to the PC and connected to the RIGOL DG5252 noise generator (Germany), which simulated the operation of the RES complex.

For the experiment, a training sample was used, which contains data on the state of the electronic environment of the group. 5000 observations from this sample were used for experiments. The training sample contained 3,000 observations, the test sample contained 2,000 observations. 


\section{The results of research on the RES assessment and forecasting in intelligent decision making support systems}

3. 1. Mathematical problem statement for the development of methods for RES assessing and forecasting in intelligent decision making support systems

Let's perform a mathematical formulation of the problem of developing methods for the RES assessing and forecasting in intelligent decision making support systems.

Setting the objectives.

Given: radio communication devices settings $\Psi=\left\{\psi_{i}\right\}, i=\overline{1, m}: S$ is the number of transmitting antennas, $V$ is the number of receiving antennas, $v_{\text {tran }}$ is the operating frequency tuning speed, $K_{s u b}$ is the number of frequency subchannels, $M$ is the type of signal modulation and its positioning; $K_{\text {change }}$ is the number of options for the speed of adjustment of the operating frequency, $P_{S}$ is the useful signal power, $\Delta F_{s}$ is the signal frequency band, $h_{n}^{2}$ is the signal-to-noise ratio, $H(t)$ is the transmission characteristic of the communication channel, $R$ is the coding speed; $v_{i}$ is the speed of information transmission by radio communication.

It is necessary: to identify the radio communication devices, its type, parameters and operation mode, the current position of the frequency-time parcel and to predict the further position of the frequency-time parcel of its signal with a given accuracy at the time with minimum identification time $t_{i d} \rightarrow \min$.

Restrictions and assumptions according to the limitations and assumptions given in the previous section of the research.

The task of identifying the radio communication devices, its type, parameters and mode of operation and predicting the future position of the frequency-time sending of its signal is reduced to a typical optimization problem. The system of equations for solving the optimization problem is the following:

$$
\left\{\begin{array}{l}
\Omega(t)=F_{1}\left(h_{n}^{2}, S, V, M, K_{\text {sub }}, P_{s}\right) \rightarrow \max \\
t_{i d}=F_{n}\left(h_{n}^{2}, S, V, M, K_{\text {change }}, P_{S}\right) \rightarrow \min
\end{array}\right.
$$

Since the process of analysis of the electronic environment is a dynamic process, let's write the functional (1) RES state analysis and prediction for a dynamic system in the form of a multidimensional time series:

$$
\forall t \in\{1, \ldots, T, \ldots\} \Psi_{t}=\left\{\begin{array}{c}
\psi_{1}^{(t)} F_{1}\left(\varphi_{1,1}\left(s_{1}^{(t-1)}, \ldots, s_{1}^{\left(t-L_{1}^{1}\right)}\right), \varphi_{1, N}\left(s_{N}^{(t-1)}, \ldots, s_{N}^{\left(t-L_{1}^{N}\right)}\right)\right) \times 1_{1}, \\
\psi_{2}^{(t)} F_{2}\left(\varphi_{2,1}\left(\psi_{1}^{(t-1)}, \ldots, \psi_{1}^{\left(t-L_{2}^{1}\right)}\right), \varphi_{2, N}\left(\psi_{N}^{(t-1)}, \ldots, \psi_{N}^{\left(t-L_{2}^{N}\right)}\right)\right) \times 1_{2}, \\
\ldots \\
\psi_{N}^{(t)} F_{N}\left(\varphi_{N, 1}\left(\psi_{1}^{(t-1)}, \ldots, \psi_{1}^{\left(t-L_{N}^{1}\right)}\right), \varphi_{N, N}\left(\psi_{N}^{(t-1)}, \ldots, \psi_{N}^{\left(t-L_{N}^{N}\right)}\right)\right) \times 1_{N},
\end{array}\right.
$$

where $\Psi_{t}$ is the multidimensional time series; $\Psi_{t}=\left(\psi_{1}^{(t)}, \psi_{2}^{(t)}, \ldots, \psi_{N}^{(t)}\right)$ is the time slice of the RES analysis state is presented in the form of a multidimensional time series at the $t$-th moment of time, namely the parameters of radio communication devices $\Psi=\left\{\psi_{i}\right\}$ at a time with a given accuracy $\Omega(t) ; \psi_{j}^{(t)}$ is the value of the $j$-th component of the multidimensional time series at the $t$-th time; $L_{j}^{i}$ is the maximum value of the time delay of the $i$-th component relative to the $j$-th; $\varphi_{i j}$ is the operator to take into account the interaction between the $i$-th and $j$-th component of the multidimensional time series; $F_{i}$ is the conversion to obtain $s^{(t)}, i=1, \ldots, N ; N$ is the number of components of the multidimensional time series; $\mathrm{l}$ is the operator to take into account the degree of awareness of the RES state.

From expression (2) it is possible to conclude that the expression allows to describe the processes of RES analysis, taking into account time delays. Delays are required to collect, process and summarize information on the RES state, as well as to take into account the degree of awareness 
of the RES state. Also, this expression (2) allows to describe processes that have both quantitative and qualitative units.

Given the above, it is proposed to develop a methodology for assessing and forecasting the electronic environment in intelligent decision making support systems in order to reduce the time to identify the parameters of radio communication devices with a given accuracy.

\section{2. Development of methods for assessing and forecasting the electronic environment} in intelligent decision making support systems

The improved method of assessment and forecasting of the electronic environment in intelligent decision making support systems consists of the following sequence of actions (Fig. 1):

1. Enter the source data. At this stage, the initial data available on RES are entered. The basic model of the RES state of the region with typical parameters of radio electronic devices and radio electronic warfare devices is initialized $[14,15]$.

2. Identify the factors and relationships between them. At this stage, there is an analysis of the problem, determining the purpose and objectives of the analysis and forecasting the RES state and cognitive structuring of available information about the RES state.

$$
\psi_{i(\text { norm })}^{\left(t-l_{i}^{j}\right)}=\frac{\psi_{i}^{\left(t-l_{i}^{j}\right)}-\psi_{i(\min )}}{\psi_{i(\max )}-\psi_{i(\min )}}, l_{i}^{j}=0, \ldots, L_{i}^{j},
$$

where $\psi_{i(\text { norm })}^{\left(t-l_{i}^{j}\right)}$ is the normalized value; $\psi_{i(\max )}, \psi_{i(\min )}$ is the maximum and minimum values, respectively.

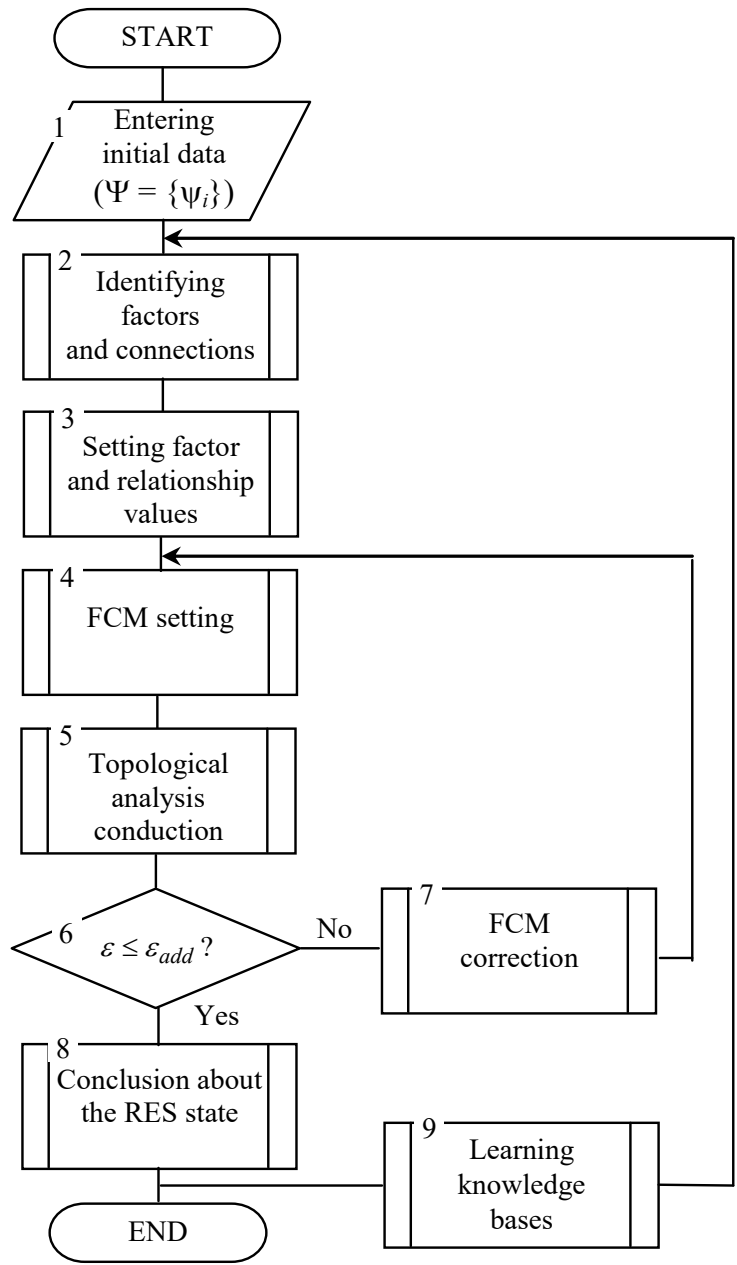

Fig. 1. Algorithm for implementing methods of RES analysis and forecasting 
3. Setting the values of factors and relationships between them.

3. 1. Processing of initial data.

The values of the parameters of the vertices $x_{v_{i}}, i=\overline{1, h}$ ( $h$ is the number of factors) can be represented as: numbers that differ in units and order of magnitude; intervals $x_{v_{i}}=\left[x_{v_{i} 1}, x_{v_{i}} 2\right]$, where $x_{v_{i} 1}, x_{v_{i} 2}$ are the minimum and maximum value of vertices and verbal descriptions; fuzzy triangular numbers $x_{v_{i}}=\left[x_{v_{i}} 1, x_{v_{i}}, x_{v_{i}} 3\right]$, where $x_{v_{i}} 1, x_{v_{i} 2}, x_{v_{i} 3}$ are the minimum, most expected and maximum value of the vertices; fuzzy trapezoidal numbers $x_{v_{i}}=\left[x_{v_{i}}, x_{v_{i} 2}, x_{v_{i} 3}, x_{v_{i}}\right]$, where $x_{v_{i} 1}, x_{v_{i} 2}$ are the pessimistic and optimistic estimates of the boundaries of the intervals, $x_{v_{i} 3}, x_{v_{i}} 3$ are the interval of the most possible estimate.

3. 2. Normalization of values of parameters of vertices presented in the form of intervals, fuzzy numbers.

3. 3. Normalization of values of relations between vertices, presented in the form of intervals of fuzzy numbers.

3. 4. Structuring the values of connections between vertices.

4. Fuzzy Cognitive Maps (FCM) construction.

FCM of the RES state is to establish structural relationships (in the form of time lags) between the FCM concepts, weighted by the fuzzy values $w_{i j}^{\left(t-l_{i}^{j}\right)}$ of their influence on each other. In this work, modified ANFIS-type models (Adaptive Neuro-Fuzzy Inference System) are proposed as $F S_{i}$ FCM that implement fuzzy temporal transformations of $F_{i}$ :

$$
\tilde{\psi}_{j}^{\prime\left(t-l_{i}^{j}\right)}=\left(w_{i j}^{\left(t-l_{i}^{j}\right)} \mathrm{T} \tilde{\psi}_{j}^{\left(t-l_{i}^{j}\right)}\right), l_{i}^{j}=0, \ldots, L_{i}^{j},
$$

where $T$ is the T-norm operation.

Then there is an aggregation based on the operation of the T-norm of the truth degrees of the rules preconditions:

$$
\begin{aligned}
& \alpha_{p}=\min \mu_{\tilde{L}}\left(\tilde{\psi}_{1}^{\prime(t-1)}\right), \mu_{\tilde{L}}\left(\tilde{\psi}_{3}^{\prime(t-3)}\right), \\
& \mu_{\tilde{M}}\left(\tilde{\psi}_{4}^{\prime(t-3)}\right), \mu_{\tilde{M}}\left(\tilde{\Psi}_{5}^{\prime(t-3)}\right), \mu_{\tilde{H}}\left(\tilde{\psi}_{1}^{\prime(t-3)}\right) .
\end{aligned}
$$

Then, activate the conclusion of the relevant rules in accordance with the degrees of truth of their assumptions based on the operation of implication (here, the implications of Mamdani operation of min-activation) $[16,17]$ :

$$
\mu_{\tilde{M}}\left(\tilde{\psi}_{1}^{\prime(t)}\right)=\min \left(\alpha_{p}, \tilde{M}\right)
$$

Then, the max-disjunction operation is performed, accumulating activated conclusions of all model rules:

$$
\tilde{s}_{1}^{(t)}=\max \left(\mu_{\tilde{M}}\left(\tilde{s}_{1}^{\prime(t)}\right), \ldots, \mu_{\tilde{M}}\left(\tilde{s}_{1}^{\prime(t)}\right), \ldots, \mu_{\tilde{H}}\left(\tilde{s}_{1}^{\prime(t)}\right)\right)
$$

Next is the normalization, storage and output of fuzzy values of the output variables of the model required for FCM with time delays:

$$
\tilde{\Psi}_{1(\text { norm })}^{(t)}=Z^{0}\left(\tilde{\Psi}_{1}^{(t-1)}\right), \tilde{\psi}_{1(\text { norm })}^{(t-2)}=Z^{-1}\left(\tilde{\Psi}_{1}^{(t-1)}\right) .
$$

5. Topological analysis of the FCM structure.

The procedure of topological analysis of the FCM structure consists of the following sequence of actions:

Step 5. 1. Enter the values of the relationships between the FCM vertices.

Step 5. 2. If the values of the connections between the vertices are presented in the form of verbal descriptions are followed, then go to step 5. 3, and if not then go to step 5. 4.

Step 5. 3. Structuring the values of the relationships between the vertices. 
Step 5. 4. If the condition that the values of the relationships between the vertices are represented as intervals of fuzzy numbers is fulfilled, then let's move on to step 5. 5; if the condition is not fulfilled, so the values of the connections between the vertices are represented by numbers from the interval $w_{i j} \in[1,1]$ then let's proceed to step 5. 6 .

Step 5. 5. Normalize the values of the relationships between the vertices, which are represented as intervals, fuzzy numbers.

Action 5. 6. FCM construction in the form of a matrix of relations.

Step 5. 7. Go from undefined values to «-1», «0» and «1». To apply the topological analysis of the FCM structure, it is recommended to convert the obtained normalized interval values of connections between vertices as follows:

1) if the normalized values are in the interval $[-1,0)$, then it is a minus «one»;

2) if the normalized value is in the interval $(0,0.5)$ it means «zero»;

$3)$ if the normalized value is in the interval $[0,5,1]$ it means «one».

Step 5. 8. Construction of a matrix of relations consisting of «-1», «0» and «1».

Step 5. 9. Calculation of the dimension of the simplexes of the complex $K_{x}(Y ; \lambda)$ Firstly, the units in each $i$-th line are counted, $i=1,2, \ldots, m$, and then the dimension of the simplexes of the complex $K_{x}(Y ; \lambda)$ is calculated.

$$
q=q^{(i)}=\sum_{j=1}^{m} \lambda_{i j}-1
$$

Step 5. 10. Calculation of the simplexes dimension of the complex $K_{y}\left(X ; \lambda^{*}\right)$.

Firstly, the units in each $j$-th column are counted, and then the dimension of the simplexes of the $K_{y}\left(X ; \lambda^{*}\right)$ complex is calculated.

$$
q=q^{(j)}=\sum_{j=1}^{m} \lambda_{i j}-1 .
$$

Step 5. 11. Matrix transformation is performed.

The transformation of the matrix $\Lambda$ into ${ }^{(1)} \Lambda$ - ordering the terms from top to bottom is carried out according to the following rule:

$$
q_{1}^{(i)}>q_{2}^{(i)}>q_{3}^{(i)} \ldots>0>-1 .
$$

The transformation of the matrix ${ }^{(1)} \Lambda$ into ${ }^{(2)} \Lambda-$ the ordering of $j$-columns from left to right is carried out by the rule:

$$
q_{1}^{(j)}>q_{2}^{(j)}>q_{3}^{(j)} \ldots>0>-1
$$

Step 5. 12. Simplicial complexes are constructed.

The construction of the $K_{x}(Y ; \lambda)=\left\{\delta_{q}^{\left(v_{i}\right)}\right\}$ complex is a sequence of $\delta_{q}^{\left(v_{i}\right)}$ simplexes, which is ordered by the rule (10) of decreasing their dimension.

The construction of the $K_{x}(Y ; \lambda)=\left\{\delta_{q}^{\left(v_{i}\right)}\right\}$ complex is a sequence of $\delta_{q}^{\left(v_{i}\right)}$ simplexes, which is ordered by the rule (11) of decreasing their dimension.

Step 5. 13. Determine the first structural vector on the matrix ${ }^{(2)} \Lambda$ of the $K_{x}(Y ; \lambda)$ complex.

Determination of the matrix ${ }^{(2)} \Lambda$ of the first structural vector $Q_{x}=\left\{Q_{\operatorname{dim} K}, \ldots, Q_{g}, Q_{1}, Q_{0}\right\}$ of the complex $K_{x}(Y ; \lambda)$ is as follows. For each dimension $q^{(i)}$ the number of simplexes in each equivalence class $Q_{q}$ is set by the rule: if at least one unit of the $i$-string is not included in the previous strings $i-1, i-2, \ldots, 1$, then the simplex corresponding to this line forms a separate equivalence class.

Step 5. 14. The first structural vector on the matrix ${ }^{(2)} \Lambda K_{y}\left(Y ; \lambda^{*}\right)$ is determined. For each dimension $q^{(j)}$ the number of simplexes in each equivalence class $Q_{q}$ is set by the rule: if at least one unit of the $j$-th column is not included in the previous columns $j-1, j-2, \ldots, 1$, then the simplex corresponding to this column forms separate equivalence class. 
6. ANN (artificial neural networks) training.

In this procedure, the $\mathrm{ANN}$ is taught using the evolving method developed by the authors in the work $[2,18]$. This method differs from the known ones because it allows training not only synaptic scales, but also the parameters of the membership function together with the ANN architecture.

7. Forecasting the RES state.

Multidimensional analysis and prediction of the complex system/process state is performed on the basis of structurally and parametrically configured FCM and can be performed in the following modes:

- firstly, direct multidimensional prediction of the complex system/process state for the $t$-th time, so the calculation of the values of the output variable models $F S_{i}, i=1, \ldots, N$ on each given by the respective sets of values of the input variables of these models;

- secondly, self-development and forecast assessment of changes in the state of a complex system/process, in which modeling the dynamics of state change is carried out from a situation given the initial values of all concepts FCM, in the absence of external influences;

- thirdly, the development and forecast assessment of changes in the state of a complex system/process in which modeling the dynamics of state change is carried out in a given situation. The situation is given by the initial knowledge of all the concepts of FCM, with external influence on the meaning of the concepts and/or on the relationship of influence between the FCM concepts.

\section{Discussion}

Modeling and evaluation of the effectiveness of the proposed method was performed in accordance with the conditions of modeling with the involvement of tools, which are described in detail in section 2 of this research. Fig. 2, 3 show the simulation of the duel conflict of radio communication and electronic warfare devices. Moreover, the proposed method of estimation and prediction was used in the means of electronic warfare at the stage of identification of the radio communication devices and prediction of the position of the signal in the frequency-time plane.

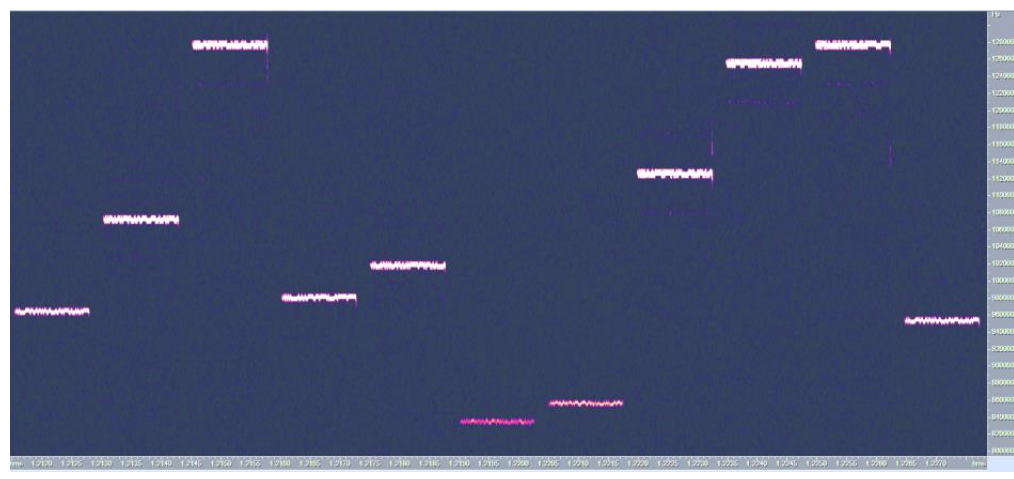

$a$

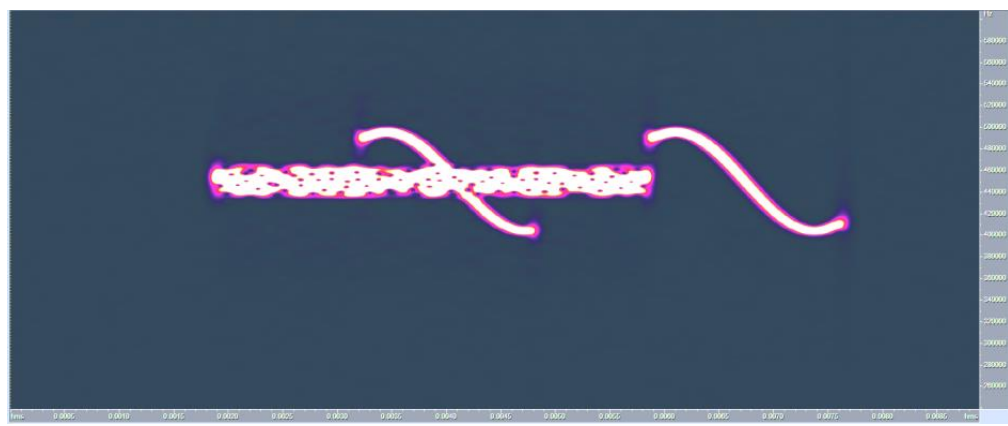

$b$

Fig. 2. Operation of 5 transceivers according to the specified method and two noise generators RIGOL DG5252: $a$ - the general representation of FHSS; $b$ - the detailed representation of the influence of interference on the FHSS systems during modeling 


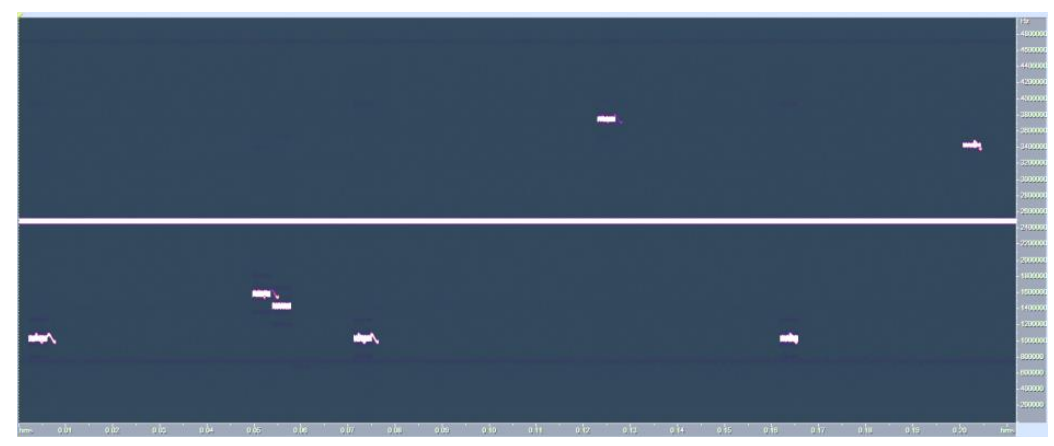

Fig. 3. Demonstration of the effectiveness of the use of the training and forecasting procedure using the proposed methodology

Fig. 2, 3 shows, however, when using the procedure of training and prediction, the number of affected frequency elements increased by an average of $20 \%$.

The square root of the standard error was used as a criterion for the quality of forecasting.

A multilayer perceptron (MLP), a radial-base neural network (RBNN), and an evolving artificial neural network were used to compare the quality of the prediction.

The forecasting results for different systems are presented in Table. 1.

Table 1

Forecasting results for different systems

\begin{tabular}{lcccc}
\hline \multicolumn{1}{c}{ System name } & $\begin{array}{c}\text { Number of customizable } \\
\text { parameters }\end{array}$ & $\begin{array}{c}\text { RMSE } \\
\text { (educational) }\end{array}$ & $\begin{array}{c}\text { RMSE } \\
\text { (test) }\end{array}$ & Time, s \\
\hline Multilayer perceptron & 51 & 0.1058 & 0.1407 & 0.1081 \\
Radial-basic neural network & 21 & 0.1066 & 0.2155 & 0.1081 \\
Evolving cascade system with neo-phase nodes & 20 & 0.0784 & 0.1081 & 0.1081
\end{tabular}

These results can be seen from the results in the last terms of Tables 1, 2 as the difference of the Xi-Beni index.

The main advantages of the proposed evaluation methodology are:

- it has a flexible hierarchical structure of indicators, which allows to reduce the task of multi-criteria evaluation of alternatives to one criterion or to use a vector of indicators for selection;

- unambiguity of the received estimation of RES condition;

- wide scope of use (support and decision-making systems);

- simplicity of mathematical calculations;

- it does not accumulate a learning error;

- ability to adapt the system of indicators in the course of work;

- learning not only the synaptic weights of the artificial neural network, but also the type and parameters of the membership function;

- learning the architecture of artificial neural networks;

- calculation of data for one epoch without the need to store previous calculations;

- possibility of synthesizing the optimal structure of FCM.

It should be noted that the proposed training procedure showed the best PC (partition coefficient, PC) in comparison with EFCM and the best result in time compared to FCM. The study showed that this training procedure provides on average 10-18\% higher learning efficiency of artificial neural networks and does not accumulate errors during training (Table 1).

The disadvantages of the proposed method include:

- loss of informativeness in assessing the RES state due to the construction of the membership function. This loss of information can be reduced by choosing the type of membership function in the practical implementation of the proposed methodology in support and decision-mak- 
ing systems. The choice of the type of membership function depends on the computing resources of a particular electronic computing device;

- lower accuracy of estimation on separately taken parameter of estimation of RES condition;

- lower accuracy of estimation in comparison with other estimation methods.

This technique will allow to:

- assess the RES state;

- identify effective measures to improve management efficiency;

- increase the speed of RES assessment;

- reduce the use of computing resources and decision-making support systems.

Restrictions about the use of this technique include:

- the need to form and constantly update the knowledge base;

- RES assessment status occurs with delays caused by the need for calculations and delays arising from the remoteness of the RES collection and analysis from each other;

- for the correct start of work it is necessary to have a standard knowledge base of RES of the work region;

- the method should not be used in RES one-parameter assessment;

- the use of the knowledge base training procedure requires correct long test and training sequences for each type of radio sources.

The analysis of the method showed that the computational complexity is $15-25 \%$ less, compared with the methods used to assess the effectiveness of decisions, which are presented in Table 1. This research is a further development of research aimed at developing methodological principles for improving the efficiency of information and analytical support, published earlier $[1,2,5]$.

Areas of further research should be aimed at reducing computational costs in the processing of various types of data in special purpose systems.

\section{Conclusions}

Mathematical formulation of the problem of developing methods for RES assessment and forecasting in intelligent decision making support systems. This allows to describe the processes that take place in intelligent decision making support systems in solving problems of RES analysis and forecasting. As a criterion for the effectiveness of this technique, the accuracy of analysis and prediction of the RES state when the restrictions on the time of identification. The statement of the research task shows that the process of analysis and forecasting is a complex hierarchical process, which is reduced to solving the problem of multi-criteria analysis of alternatives.

That is why, in the course of this research, a methodology for RES assessing and forecasting in intelligent decision making support systems has been developed, which allows to:

- conduct a multidimensional RES analysis and forecasting in conditions of uncertainty;

- provide a forecast estimate in conditions of non-stochastic uncertainty, nonlinearity of mutual influence, partial inconsistency and significant interdependence of components of a multidimensional time series;

- train artificial neural networks for intelligent decision making support systems;

- conduct a topological analysis of the FCM structure;

- process the values of connections between factors, which are presented in the form of numbers, verbal descriptions, and also allows to substantiate the choice of target and control vertices of FCM in the conditions of uncertainty of initial data.

An example of using the proposed method on the example of assessment and forecasting the RES state. This example showed an increase in data processing efficiency at the level of $15-25 \%$ due to the use of additional advanced procedures.

\section{References}

[1] Kuchuk, N., Mohammed, A. S., Shyshatskyi, A., Nalapko, O. (2019). The method of improving the efficiency of routes selection in networks of connection with the possibility of self-organization. International Journal of Advanced Trends in Computer Science and Engineering, 8 (1). 1-6. Available at: https://www.elibrary.ru/item.asp?id=41636925 
[2] Sova, O., Turinskyi, O., Shyshatskyi, A., Dudnyk, V., Zhyvotovskyi, R., Prokopenko, Y. et. al. (2020). Development of an algorithm to train artificial neural networks for intelligent decision support systems. Eastern-European Journal of Enterprise Technologies, 1 (9 (103)), 46-55. doi: https://doi.org/10.15587/1729-4061.2020.192711

[3] Zagranovskaya, A. V., Eyssner, Yu. N. (2017). Simulation scenarios of the economic situation based on fuzzy cognitive maps. Modern economics: problems and solutions, 10 (94), 33-47. doi: https://doi.org/10.17308/meps.2017.10/1754

[4] Simankov, V. S., Putyato, M. M. (2013). Issledovanie metodov kognitivnogo analiza. Perspektivy razvitiya informatsionnyh tekhnologiy, 13, 31-35.

[5] Onykiy, B., Artamonov, A., Ananieva, A., Tretyakov, E., Pronicheva, L., Ionkina, K., Suslina, A. (2016). Agent Technologies for Polythematic Organizations Information-Analytical Support. Procedia Computer Science, 88, 336-340. doi: https://doi.org/ 10.1016/j.procs.2016.07.445

[6] Ko, Y.-C., Fujita, H. (2019). An evidential analytics for buried information in big data samples: Case study of semiconductor manufacturing. Information Sciences, 486, 190-203. doi: https://doi.org/10.1016/j.ins.2019.01.079

[7] Çavdar, A. B., Ferhatosmanoğlu, N. (2018). Airline customer lifetime value estimation using data analytics supported by social network information. Journal of Air Transport Management, 67, 19-33. doi: https://doi.org/10.1016/j.jairtraman.2017.10.007

[8] Shyshatskyi, A., Zvieriev, O., Salnikova, O., Demchenko, Ye., Trotsko, O., Neroznak, Ye. (2020). Complex Methods of Processing Different Data in Intellectual Systems for Decision Support System. International Journal of Advanced Trends in Computer Science and Engineering, 9 (4), 5583-5590. doi: https://doi.org/10.30534/ijatcse/2020/206942020

[9] Narendra, K. S., Parthasarathy, K. (1990). Identification and control of dynamical systems using neural networks. IEEE Transactions on Neural Networks, 1 (1), 4-27. doi: https://doi.org/10.1109/72.80202

[10] Zhou, X., Zhuge, Q., Qiu, M., Xiang, M., Zhang, F., Wu, B. et. al. (2018). Bandwidth variable transceivers with artificial neural network-aided provisioning and capacity improvement capabilities in meshed optical networks with cascaded ROADM filtering. Optics Communications, 409, 23-33. doi: https://doi.org/10.1016/j.optcom.2017.09.021

[11] Bodyanskiy, E., Strukov, V., Uzlov, D. (2017). Generalized metrics in the problem of analysis of multidimensional data with different scales. Scientific Works of Kharkiv National Air Force University, 3 (52), 98-101. Available at: http://www.hups.mil. gov.ua/periodic-app/article/17760

[12] Zhou, S., Yin, Z., Wu, Z., Chen, Y., Zhao, N., Yang, Z. (2019). A robust modulation classification method using convolutional neural networks. EURASIP Journal on Advances in Signal Processing, 2019 (1). doi: https://doi.org/10.1186/s13634-019-0616-6

[13] Malik, S., Kumar, S. (2017). Optimized Phase Noise Compensation Technique using Neural Network. Indian Journal of Science and Technology, 10 (5), 1-6. doi: https://doi.org/10.17485/ijst/2017/v10i5/104348

[14] Zhang, D., Ding, W., Zhang, B., Xie, C., Li, H., Liu, C., Han, J. (2018). Automatic Modulation Classification Based on Deep Learning for Unmanned Aerial Vehicles. Sensors, 18 (3), 924. doi: https://doi.org/10.3390/s18030924

[15] Zhyvotovskyi, R., Shyshatskyi, A., Petruk, S. (2017). Structural-semantic model of communication channel. 2017 4th International Scientific-Practical Conference Problems of Infocommunications. Science and Technology (PIC S\&T). doi: https://doi.org/ 10.1109/infocommst.2017.8246454

[16] Harding, J. L. (2013). Data quality in the integration and analysis of data from multiple sources: some research challenges. The International Archives of the Photogrammetry, Remote Sensing and Spatial Information Sciences, XL-2/W1, 59-63. doi: https://doi.org/10.5194/isprsarchives-xl-2-w1-59-2013

[17] Gödri, I., Kardos, C., Pfeiffer, A., Váncza, J. (2019). Data analytics-based decision support workflow for high-mix low-volume production systems. CIRP Annals, 68 (1), 471-474. doi: https://doi.org/10.1016/j.cirp.2019.04.001

[18] Tymchuk, S. (2017). Methods of Complex Data Processing from Technical Means of Monitoring. Path of Science, 3 (3), 4.1-4.9. doi: https://doi.org/10.22178/pos.20-4

How to cite: Sova, O., Shyshatskyi, A., Salnikova, O., Zhuk, O., Trotsko, O., Hrokholskyi, Y. (2021). Development of a method for assessment and forecasting of the radio electronic environment. EUREKA: Physics and Engineering, 4, 30-40. doi: https://doi.org/10.21303/2461-4262.2021.001940 